\title{
Optimization and Improvement in Robot-Based Assembly Line System by Hybrid Genetic Algorithm
}

\author{
Lin Lin Student Member (Waseda University, lin@ruri.waseda.jp) \\ Mitsuo Gen Member (Waseda University) \\ Jie Gao Non-member (Xian Jiaotong University)
}

Keywords : balancing, improvement, genetic algorithms, local search, neighborhood structure, robotic assembly line

An assembly line system is a manufacturing process in which interchangeable parts are added to a product in a sequential manner to produce a finished product. In the past decades, robots have been extensively applied in assembly lines as called robotic assembly lines. An assembly robot can work 24 hours a day without worries or fatigue. Goals for implementation of robotic assembly lines include high productivity, quality of product, manufacturing flexibility, safety, decreasing demand of skilled labor, and so on. Usually, specific tooling is developed to perform the activities needed at each station. Such tooling is attached to the robot at the station. In order to avoid the time waste required for tool change, the design of the tooling can take place only after the line has been balanced. Different robot types may exist at the assembly facility. Each robot type may have different capabilities and efficiencies for various elements of the assembly tasks. Hence, to allocate the best fitting robot for each station is critical for the performance of robotic assembly lines.

In this paper, we also consider a hybrid GA approach for solving rALB-2 problems. We not only focus on the design of GA to minimize the cycle time of the assembly systems, but also hammer at the key point of improvement of assembly system depended on the final result analysis. We propose a hybrid genetic algorithm (hGA) to solve the rALB with minimizing the cycle time. The GA uses partial representation technique, that is, the coding space only contains a part of the feasible solutions, in which the optimal solution is included. New crossover and mutation operators are also developed to adapt to the specific chromosome structure and the nature of the problem. In order to strengthen the search ability, a local search procedure works under the framework of GA, this only investigates the neighbor solutions that have possibilities to improve the solutions of current population.

A feasible solution and the best solution for a simple example are shown in Fig. 1 and Fig. 2.

We can see the best solution got the balancing for the line by comparing with the feasible solution. In the real world, the assembly line is not just for producing one unit of the product. It should produce several units. So we give the Gantt chart with 3

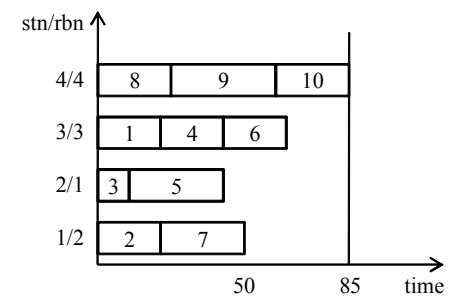

Fig. 1. Balancing chat of the feasible solution units for analyzing the solution just like Fig. 3, which is shown at the beginning. We can see the solution reduce the waiting time for the line by comparing with the feasible solution from Fig. 4. It also means the better solution got the balancing for the assembly line.

The performance of proposed method is validated through simulation experiments. The simulation shows that our algorithm is computationally efficient and effective to find the best solution. The solution obtained by our algorithm outperforms the results from previous works.

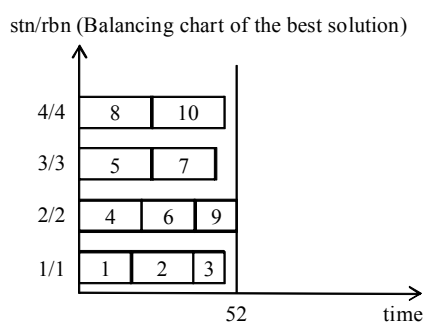

Fig. 2. The balancing chat of the best solution

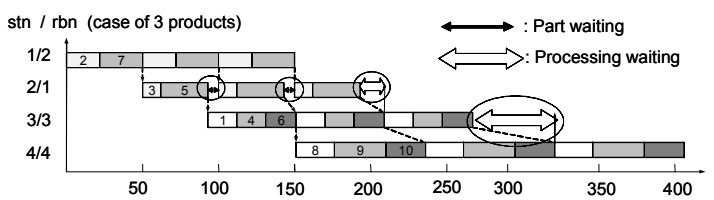

Fig. 3. Gant chat for producing 3 units of the feasible solution

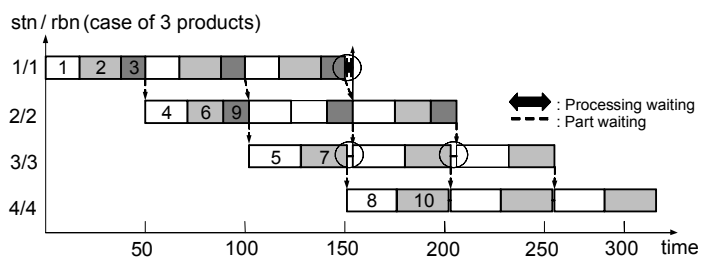

Fig. 4. Gant chat of the best solution for producing 3 units

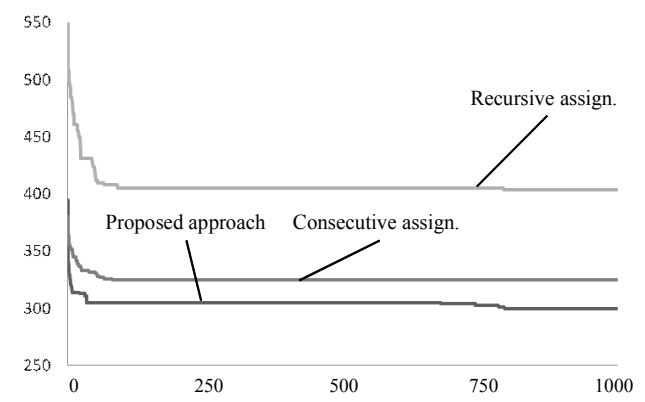

Fig. 5. Evolutionary process of propose approach and Levitin et al.'s two algorithms 


\title{
Optimization and Improvement in Robot-Based Assembly Line System by Hybrid Genetic Algorithm
}

\author{
Lin Lin* Student Member \\ Mitsuo Gen* Member \\ Jie Gao** Non-member
}

\begin{abstract}
In the real world, there are a lot of scenes from which the product is made by using the robot, which needs different assembly times to perform a given task, because of its capabilities and specialization. For a robotic assembly line balancing (rALB) problem, a set of tasks have to be assigned to stations, and each station needs to select one robot to process the assigned tasks. In this paper, we propose a hybrid genetic algorithm (hGA) for solving this problem. In the hGA, we use new representation method. Advanced genetic operators adapted to the specific chromosome structure and the characteristics of the rALB problem are used. In order to strengthen the search ability, a local search procedure is integrated under the framework the genetic algorithm. Some practical test instances demonstrate the effectiveness and efficiency of the proposed algorithm.
\end{abstract}

Keywords : balancing, improvement, genetic algorithms, local search, neighborhood structure, robotic assembly line

\section{Introduction}

An assembly line system is a manufacturing process in which interchangeable parts are added to a product in a sequential manner to produce a finished product. Assembly lines are the most commonly used method in a mass production environment, because they allow the assembly of complex products by workers with limited training, by dedicated machines and/or by robots. The installation of an assembly line is a long-term decision and usually requires large capital investments. Therefore, it is important that an assembly line is designed and balanced so that it works as efficiently as possible. Most of the work related to the assembly lines concentrate on the assembly line balancing (ALB). The ALB model deals with the allocation of the tasks among stations so that the precedence relations are not violated and a given objective function is optimized.

Since the ALB model was first formulated by Helgeson et al. $(1954)^{(1)}$, mnay versions of ALB arise by varying the objecitve function $^{(2)}$ : Type-F is an objective independent problem which is to establish whether or not a feasible line balance exists. Type-1 and Type-2 have a dual relationship; the first one tries to minimize the number of stations for a given cycle time, and the second one tries to minimize the cycle time for a given number of stations . Type-E is the most general problem version, which tries to maximize the line efficiency by simultaneously minimizing the cycle time and a number of stations . Finally, Type-3, 4 and 5 correspond to maximization of workload smoothness, maximization of work relatedness and multiple objectives with Type-3 and Type-4, respectively ${ }^{(3)}$. Furthermore, most versions of above models are NP-hard ${ }^{(4)}$.

Recently, genetic algorithm (GA) and other evolutionary algorithms (EAs) have been successfully applied in a wide variety of ALB problems. Falkenauer and Delchambre ${ }^{(5)}$ were the first to solve ALB with GAs. Following application of GAs for solving

* Waseda University, IPS

** Xian Jiaotong University
ALB model was studied by many researchers, e.g., Anderson and Ferris $^{(6)}$, Rubinovitz and Levitin ${ }^{(7)}$, Gen et al. ${ }^{(8)}$, Bautista et al. ${ }^{(9)}$, Sabuncuoglu et al. ${ }^{(10)}$, Goncalves and Almeida ${ }^{(11)}$, Brown and Sumichrast ${ }^{(12)}$, and Nearchou ${ }^{(13)}$. However, most of the researchers focused on the simplest version of the problem, with single objective and ignored the recent trends, i.e., mixed-model production, u-shaped lines, robotic lines and etc,

In the past decades, robots have been extensively applied in assembly lines as called robotic assembly lines. An assembly robot can work 24 hours a day without worries or fatigue. Goals for implementation of robotic assembly lines include high productivity, quality of product, manufacturing flexibility, safety, decreasing demand of skilled labor, and so on.

Usually, different robot types may exist at the assembly facility. Each robot type may have different capabilities and efficiencies for various elements of the assembly tasks. Hence, to allocate the best fitting robot for each station is critical for the performance of robotic assembly lines.

Rebinovitz and Bukchin ${ }^{(14)}$ were the first to formulate the robotic assembly line balancing problem as one of the allocating equal amounts of work to the stations on the line while assigning the most efficient robot type from the given set of available robots to each station. Their objective is to minimize the number of stations for a given cycle time. Their later work $^{(15)}$ present a branch and bound algorithm for the problem. Bukin and Tzur ${ }^{(16)}$ treat the problem with objective to minimize the total equipment cost, given a predetermined cycle time. An exact Branch and Bound algorithm is developed to solve moderate problems, meanwhile a branch-and-bound-based heuristic procedure is suggested for large problems. Tsai and $\mathrm{Yao}^{(17)}$ proposed a heuristic approach for the design of a flexible robotic assembly line which produces a family of products. Kim and Park ${ }^{(18)}$ extended the problem by considering additional constraints, i.e., due to limited space to store the parts and tools, restrictions for the joint assignment of tasks to stations are imposed. They proposed a mathematical formulation and a cutting plane procedure for this 
extension of the problem. Khouja et al. ${ }^{(19)}$ suggested statistical clustering procedures to design robotic assembly cells. Nicosia et $a l .{ }^{(20)}$ considered the problem of assigning operations to an ordered sequence of non-identical stations under the constraints of precedence relationships and a given cycle time. The objective is to minimize the cost of the stations . This formulation is very similar to the rALB problem.

The aforementioned rALB works have assumed that the cycle time is predetermined, and aimed at minimizing the number of stations or the cost of the assembly systems. Hence, these works are of the call of the Type 1 robotic assembly line balancing problem (rALB-1).

The type 2 robtic assemble line balancing problem (rALB-2) is to assign tasks to stations and to allocate robot for each station with the objective of minimum cycle time given the number of stations and available robots. Unlike general assembly lines, where actual processing times for performing activities vary considerably and optimal balance is rather of theoretical importance, the performance of robotic assembly lines depends strictly on the quality of its balance. As extended from simple ALB type-2, rALB-2 is also NP-hard.

Gao and $\mathrm{Gen}^{(21)}$ proposed a hybrid GA for solving rALB-2, in which different robots may be assigned to the assembly line tasks, and each robot needs different assembly times to perform a given task due to its capabilities and specialization. They focused on the genetic operators adapted to the specific chromosome structure and the characteristics of the rALB problems.

In this paper, we also consider a hybrid GA approach for solving rALB-2 problems. Different with prophase works ${ }^{(21)}$, we not only focus on the design of GA to minimize the cycle time of the assembly systems, but also hammer at the key point of improvement of assembly system depended on the final result analysis. We propose a hybrid genetic algorithm (hGA) to solve the rALB-2.

The rest of the paper is organized as follows. In Section 2, we formulate the mathematical model of rALB-2. The proposed GA for the problem is described in Section 3. In Section 4, introduce a local search proceudre. In Section 5, we demonstrate effectiveness comparing with different GA approaches. This paper gives the conclusion in Section 6.

\section{Robotic Assembly Line Balancing Problem}

The assembly of each product unit requires the execution of $n$ tasks (indivisible elements of work). Precedence constraints partially specify the order in which the tasks have to be performed. They can be represented by an acyclic precedence graph which contains nodes for all tasks. As mentioned above, the type II robotic assembly line balancing problem usually occurs when changes in the production process of a product take place. In this case, the robotic assembly line has to be reconfigured using the present resources (such as robots) so as to improve it efficiency for the new production process. The problem concerns how to assign the tasks to stations and how to allocate the available robots for each station in order to minimize the cycle time under the constraint of precedence relationships.

Let assembly tasks $(i=1,2, \ldots 10)$ assigned on 4 stations. Robots $\left(R_{1}, R_{2}, R_{3}, R_{4}\right)$ are to be equipped on the 4 stations. Table 1 give the successores $\operatorname{Suc}(i)$ of each task $i$, and the processing time of each task $t_{i l}$ processed by each robot. The Fig. 1 shows the precedence constrain of 10 tasks. A feasible solution are given for this example as Fig. 2 .

The balancing chart of the solution was drawn for analyzing the solution. Fig. 3 shows the idle time of the station 1, 2, 3 is very big, and it also means this line did not get the balancing for producing. In the real world, the assembly line is not just for producing one unit of the product. It should produce several units. We give the Gantt chart about 3 units for analyzing the solution just like Fig. 4.

We can see the waiting time occurs in the Fig. 4, and the waiting time means the idle time in the line. For example, the part waiting is occurred when the maximum processing time of the station, which is before the current station, is larger than the processing time of the current station. It means the current station will wait for the parts to process, which come from the anterior station. The processing waiting is occurred when the processing time of the current station is smaller than the processing time of the next station. It means the parts which were produced by the

Table 1. Data set of a simple rALB example

\begin{tabular}{|c|c|c|c|c|c|}
\hline \multirow[b]{2}{*}{ Task $i$} & \multirow[b]{2}{*}{$\operatorname{Suc}(i)$} & \multicolumn{4}{|c|}{ Task time by robot $l \quad t_{i l}$} \\
\hline & & $\mathrm{R}_{1}$ & $\mathrm{R}_{2}$ & $\mathrm{R}_{3}$ & $\mathrm{R}_{4}$ \\
\hline 1 & $\{4\}$ & 17 & 22 & 19 & 13 \\
\hline 2 & $\{4\}$ & 21 & 22 & 16 & 20 \\
\hline 3 & $\{5\}$ & 12 & 25 & 27 & 15 \\
\hline 4 & $\{6\}$ & 29 & 21 & 19 & 16 \\
\hline 5 & $\{10\}$ & 31 & 25 & 26 & 22 \\
\hline 6 & $\{8\}$ & 28 & 18 & 20 & 21 \\
\hline 7 & $\{8\}$ & 42 & 28 & 23 & 34 \\
\hline 8 & $\{10\}$ & 27 & 33 & 40 & 25 \\
\hline 9 & $\{10\}$ & 19 & 13 & 17 & 34 \\
\hline 10 & \{\} & 26 & 27 & 35 & 26 \\
\hline
\end{tabular}

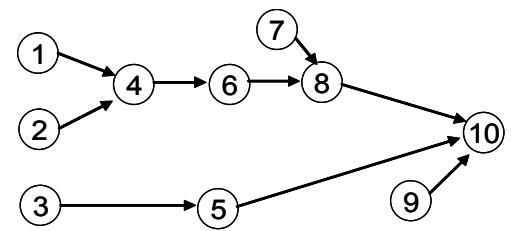

Fig. 1. Precedence graph of example problem

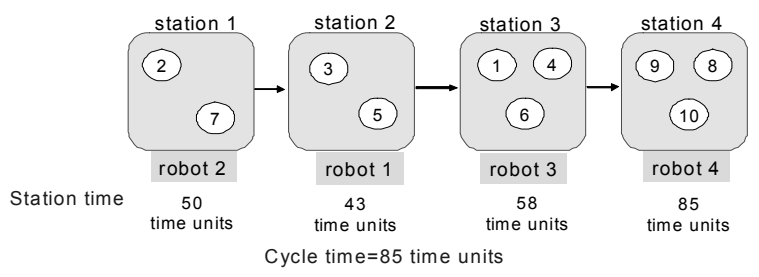

Fig. 2. The feasible solution for the example

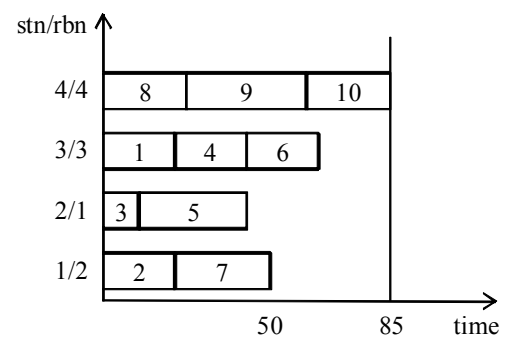

Fig. 3. Balancing chart of the feasible solution 
current station will wait for being processed by the next station. Both of the waiting time is the idle time of the assembly line. We want to reduce it by getting the balance of the line. The following assumptions are stated to clarify the setting in which the problem arises:

A1. The precedence relationship among assembly activities is known and invariable.

A2. The duration of an activity is deterministic. Activities can not be subdivided.

A3. The duration of an activity depends on the assigned robot.

A4. There are no limitations on assignment of an activities or a robot to any station. In case a task can not be processed on a robot, the assembly time of the task on the robot is set to a very large number.

A5. A single robot is assigned to each station.

A6. Material handling, loading and unloading times, as well as set-up and tool changing times are negligible, or are included in the activity times.

A7. The number of stations is determined by the number of robots, since the problem aims to maximize the productivity by using all robots at hand.

A8. The line is balanced for a single product.

The notation used in this section can be summarized as follows: Indices:
$i, j$ : index of assembly tasks, $i, j=1,2, \ldots, n$
$k$ : index of stations $, k=1,2, \ldots, m$
$l$ : index of robots, $l=1,2, \ldots, m$

Parameters:

$n$ : total number of assembly tasks

$m$ : total number of stations (robots)

$t_{i l}$ : processing time of the $i$-th task by robot $l$

pre $(i)$ : the set of predecessor of task $i$ in the precedence diagram

\section{Decision variables:}

$$
\begin{aligned}
x_{j k} & =\left\{\begin{array}{l}
1, \text { if task } j \text { is assigned to workstation } k \\
0, \text { otherwise }
\end{array}\right. \\
y_{k l} & =\left\{\begin{array}{l}
1, \text { if robot } l \text { is allocated to workstation } k \\
0, \text { otherwise }
\end{array}\right.
\end{aligned}
$$

\section{Problem Formulation:}

$$
\begin{aligned}
& \min C_{T}=\max _{1 \leq k \leq m}\left\{\sum_{i=1}^{n} \sum_{l=1}^{m} t_{i l} x_{i k} y_{k l}\right\} \\
& \text { s.t. } \quad \sum_{k=1}^{m} k x_{j k}-\sum_{k=1}^{m} k x_{i k} \geq 0, \forall i, j \in \operatorname{pre}(i) \\
& \sum_{k=1}^{m} x_{i k}=1 \\
& \sum_{l=1}^{m} y_{k l}=1
\end{aligned}
$$

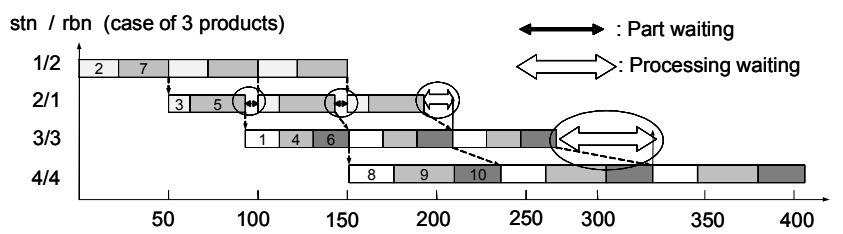

Fig. 4. Gantt chart for producing 3 units

$$
\begin{array}{ll}
\sum_{k=1}^{m} y_{k l}=1, & \forall l \cdots \\
x_{i k} \in\{0,1\} & \forall k, i \\
y_{k l} \in\{0,1\} & \forall l, k
\end{array}
$$

The objective (1) is to minimize the cycle time $\left(C_{\mathrm{T}}\right)$. Inequity (2) represents the precedence constraints. It ensures that for each pair of assembly activities, the precedent cannot be assigned to a station behind the station of the successor, if there is precedence between the two activities. Equation (3) ensures that each task has to be assigned to one station. Equation (4) ensures that each station is equipped with one robot. Equation (5) ensures that each robot can only be assigned to one station.

\section{Order-Based GA for rALB}

Genetic algorithms (GAs) are powerful and broadly applicable stochastic search and optimization techniques based on principles from evolutionary theory ${ }^{(22)}$. GA's have been applied to solve various assembly line balancing problems ${ }^{(23)-(25)}$. We divide this algorithm into 3 phases. It is as follows:

Phase 1: Deciding Task Sequence

1.1 Order encoding for task sequence: A GA's structure and parameter settings affect its performance. However, the primary determinants of a GA's success or failure are the coding by which its genotypes represent candidate solutions and the interaction of the coding with the GA's recombination and mutation operators.

A solution of the rALB problem can be represented by two integer vectors. Task sequence vector $\left(v_{1}\right)$, which contains a permutation of assembly tasks, ordered according to their technological precedence sequence, and robot assignment vector $\left(v_{2}\right)$. The solution representation method can be visually illustrated as in Fig. 6.

\begin{tabular}{|c|c|c|c|c|c|c|c|c|c|c|c|}
\hline & Locus & 1 & 2 & 3 & 4 & 5 & 6 & 7 & 8 & 9 & 10 \\
\hline Phase 1: & Task Sequence $\left(v_{1}\right)$ & 2 & 1 & 3 & 4 & 9 & 6 & 5 & 7 & 8 & 10 \\
\hline \multirow[b]{2}{*}{ se 2: } & Locus: station & & & & 2 & 3 & 4 & & & & \\
\hline & Robot assignment $\left(v_{2}\right.$ & & & & 2 & 3 & 4 & & & & \\
\hline
\end{tabular}

1.2 Reordering procedure: Considering precedence constraints, a task sequence may be infeasible since a precedent may appear before its successors. The reordering procedure will repair it into a feasible one before it is divided $m$ parts to form a solution in the phase 1 . The reordering procedure is shown in procedure 1.
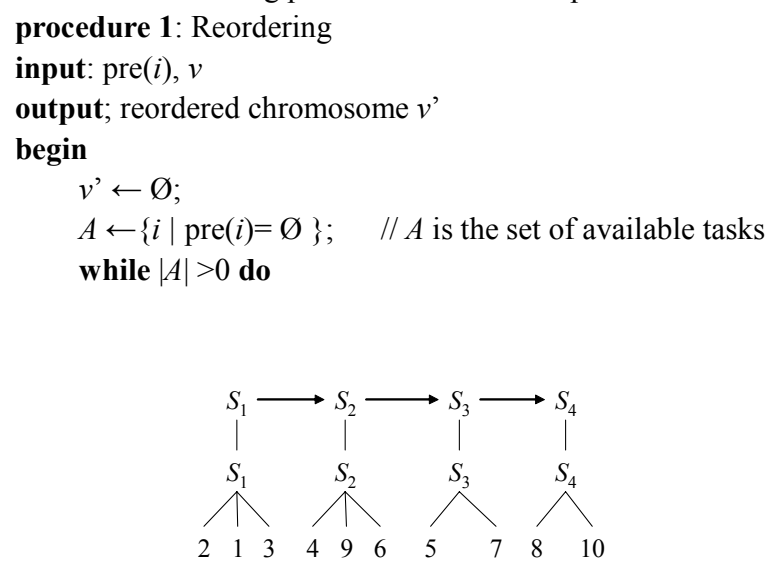

Fig. 5. Structure of example problem

Fig. 6. Solution representation of a sample problem 


$$
i^{\prime} \leftarrow \operatorname{argmin}\{v(i) \mid v(i) \in A\} ;
$$

//find the available task with highest priority

$v^{\prime} \leftarrow v^{\prime} \cup\left\{v\left(i^{\prime}\right)\right\}$;

//add the task to the reordered task sequence

$$
A \leftarrow\left\{i \mid i \notin v^{\prime} \text { and } \operatorname{pre}(i) \subseteq v^{\prime}\right\} \text {; }
$$

// update the set of available tasks

end

$$
\text { end }
$$

output reordered chromosome $v$,

1.3 Breakpoint decoding: So we use just one decoding procedure for phase 1 , and the set of decoding procedure is to generate a feasible solution based on the task sequence and robot assignment schemes which are contained in the chromosomes. Then, the breakpoint decoding procedure inserts $m$ points along the reordered task sequence vector to divide it into $\mathrm{m}$ parts, each of which corresponds to a station. The breakpoint decoding procedure consists of four main steps:

step 1: Calculate the lower bound of the cycle time $\left(C_{\mathrm{LB}}\right)$ for the solution represented by the task sequence vector and robot assignment vector;

$$
C_{L B}=\frac{1}{m} \sum_{i=1}^{n} \min _{1 \leq l \leq m}\left\{t_{i l}\right\}
$$

step 2: Find out a feasible cycle time as the upper bound of the cycle time $\left(C_{\mathrm{UB}}\right)$;

step 3: Find out the optimal cycle time via bisection method;

step 4: Partition the task sequence into $m$ parts with the optimal cycle time based on the robot assignment vector.

Here, a cycle time is said to be feasible if all the tasks can be allocated to the stations by allowing as many tasks as possible for each station under the constraint of the cycle time. The procedure to calculate the upper bound of cycle time is illustrated in procedure 2, and the bisection method to find out the optimal cycle time is shown in procedure 3 . After calculating the optimal cycle time, it is easy to generate the breakpoints on the task sequence to divide it into $m$ parts, each of which will correspond to a station, based on the robot assignment vector. Therefore, the tasks assigned for each station are determined according to a chromosome, and the cycle time can be calculated. A example of breakpoint decoding is showed in Fig. 7.

Phase 2: Assigning Robots to Station 2.1 Order encoding for robot assignment: A solution of the rALB problem can be represented by two integer vectors. In this phase, we code the robot assignment vector $\left(v_{2}\right)$, which indicates the robot assigned for each station. The solution representation method can be visually illustrated as in Fig. 6. The coding space takes an exponential growth with the length of the chromosome; therefore, even one allele saved in the chromosome can decrease the coding space significantly. An obvious advantage to intentionally omit breakpoint vector, which is used in recently research, in the chromosome is that the coding space is dramatically decreased. As a result, the speed to find the global optimal is accelerated.

2.2 Breakpoint decoding: In this phase, this robot assignment vector $\left(v_{2}\right)$ will decode into a solution with the task sequence vector $\left(v_{1}\right)$, simultaneously by the breakpoint decoding procedure. It means when the tasks are assigned into each station, the robots will be assigned into each station at the same time. The illustration of the solution is shown in Phase 1.3.

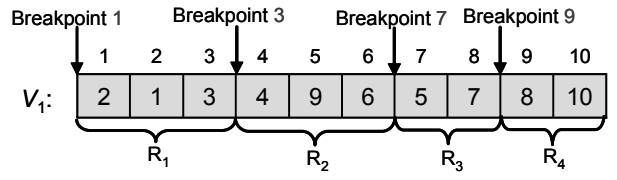

Fig. 7. Breakpoint decoding

procedure 2: Calculating Upper Bound of the Cycle Time

input: $m, n, v^{\prime}, a, C_{\mathrm{LB}}$,

output; $C_{\mathrm{UB}}$

begin

$C_{\mathrm{UB}} \leftarrow C_{\mathrm{LB}} ;$

repeat

$C_{\mathrm{UB}} \leftarrow 2 * C_{\mathrm{UB}} ; \quad / /$ multiply the upper bound by " 2 "

$i \leftarrow 0$;

for $l=1$ to $m$ do

$T \leftarrow 0$;

while $\left(t<C_{\mathrm{UB}}\right)$ and $(i<n)$ do

$i \leftarrow i+1$;

$T \leftarrow T+t_{v^{\prime}(i), a(l)} ;$

if $t>c$ then

$i \leftarrow i-1$;

until $i \geq n$;

output $C_{\mathrm{UB}}$

end

procedure 3: Bisection Method for Optimal Cycle Time

input: $m, n, v_{1}, v_{2}, C_{\mathrm{UB}}$

output: optimal cycle time $c$

begin

repeat

$C^{\prime}{ }_{\mathrm{LB}} \leftarrow C_{\mathrm{UB}} / 2$;

$C_{\mathrm{T}} \leftarrow\left(C_{\mathrm{LB}}^{\prime}+C_{\mathrm{UB}}\right) / 2$;

$i \leftarrow 0$;

for $l=1$ to $m$ do

$T \leftarrow 0 ;$

while $\left(t<C_{\mathrm{T}}\right)$ and $(i<n)$ do

$i \leftarrow i+1$;

$T \leftarrow T+t_{v^{\prime}(i), a(l)} ;$

end;

if $t>C_{\mathrm{T}}$ then

$i \leftarrow i-1$;

if $i \geq n$ then

$C_{\mathrm{UB}} \leftarrow C_{\mathrm{T}} ;$

else

$C^{\prime}{ }_{\mathrm{LB}} \leftarrow C_{\mathrm{T}} ;$

until $C_{\mathrm{UB}}-C_{\mathrm{LB}} \leq 1$

$C_{\mathrm{T}} \leftarrow C_{\mathrm{UB}}$;

output optimal cycle time $C_{\mathrm{T}}$

end

Phase 3: Making a Schedule 3.1 Creating a schedule for the assembly line: We can make a schedule based on the chromosome (in Fig. 6) as fellow:

$$
\begin{aligned}
S=\{ & \left(t_{1}, R_{1}: 0-17\right),\left(t_{2}, R_{1}: 17-38\right),\left(t_{3}, R_{1}: 38-50\right), \\
& \left(t_{4}, R_{2}: 50-71\right),\left(t_{5}, R_{3}: 102-128\right),\left(t_{6}, R_{2}: 71-89\right), \\
& \left(t_{7}, R_{3}: 128-151\right),\left(t_{8}, R_{4}: 151-176\right),\left(t_{9}, R_{2}: 89-102\right), \\
& \left.\left(t_{10}, R_{4}: 176-202\right)\right\}
\end{aligned}
$$

3.2 Drawing a Gantt chart: Firstly, we draw a balancing chart for analyzing the solution (in Fig. 8). We can see the solution got 


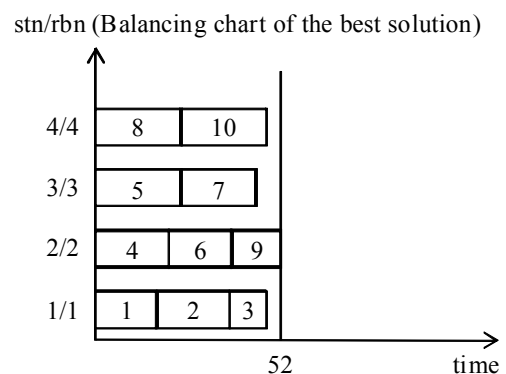

Fig. 8. The balancing chart of the best solution

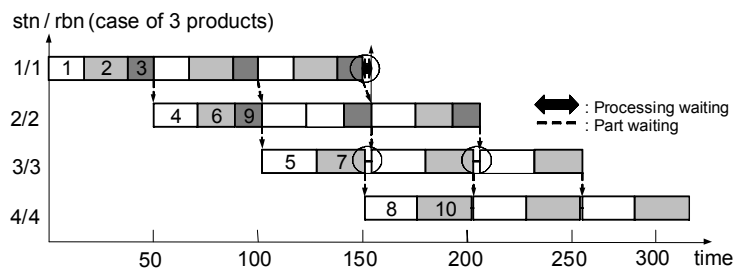

Fig. 9. Gantt chart for producing 3 units

the balancing for the line by comparing with the feasible solution from Fig. 3.

In the real world, the assembly line is not just for producing one unit of the product. It should produce several units. So we give the Gantt chart with 3 units for analyzing the solution just like Fig. 9, which is shown at the beginning. We can see the solution reduce the waiting time for the line by comparing with the feasible solution from Fig. 4. It also means the better solution got the balancing for the assembly line.

3.1 Crossover Operators In genetic algorithms, crossover recombines two chromosomes to generate a number of children. Offspring of crossover should represent solutions that combine substructures of their parental solutions ${ }^{(25)}$. In this paper, we use to kind of crossover methods: exchange crossover and mixed crossover. The detailed explanation of OX and PMX is introduced in ${ }^{(25)}$.

A. Exchange Crossover: A good schedule could be expected by exchanging task sequence and robot assignment schemes between a pair of parents. This kind of crossover is accomplished by selecting two parents and exchanging the task sequence vectors of the two parents to generate offspring.

B. Mixed Crossover: The mixed order crossover consists of two crossover methods: Order Crossover (OX) and Partial-Mapped Crossover (PMX). For the rALB chromosome, the task sequence vector and the robot assignment vector are both of the style of permutation representation. Yet, the useful features of the task sequence vector and the robot assignment vector are different.

- For the task sequence vector, the useful feature of permutation representation to represent a rALB solution is the order information among the tasks.

- For the robot assignment vector, the acting feature is the number at each allele which indicates the robot no. assigned for a specific station.

3.2 Mutation Operators In this study, two kinds of mutation operators are implemented: allele-based mutation and immigration mutation. For both the robot task sequence and robot assignment vectors, allele-based mutation randomly decides whether an allele should be mutated in a certain probability. Then, another position will be generated at random to perform exchange values with the mutated allele. In contrary to the canonical gene-by-gene mutation with very small probability at each generation, immigration mutation randomly generates one or more new members of the population from the same distribution as the initial population. This process prevents premature convergence of the population, and leads to a simple statement of convergence.

3.3 Fitness Evaluation How to evaluate the fitness of chromosome is also key issue for GAs. In the most researches, objective function is used for fitness evaluation. However, the effective fitness function have to be different function and suitable to different problems. A central problem of fitness definition is how to guide to the most promising areas from the individuals of current generation. The characteristic of ALB problems are: (1) We can calculate several different schedules with same cycle time. That means other conditions, such as idle time and total processing time are different, though the cycle time is same. For improvement of ALB, we also have to improve other conditions, not only cycle time. (2) General, the solutions with better fitness of idle time and total processing time also can create better fitness of cycle time. We define the fitness function with considering not only the objective function (cycle time), but also idle time and total processing time. It is useful to guide to the most promising areas depending on the wide conditions from the individuals of current generation. we first define the fitness value $f_{1}(v)$ as the reciprocal of the cycle time $C_{\mathrm{T}}(v)$ of the solution candidate $v$, the fitness values $f_{2}(v)$ as number of critical stations $n_{c}$ and the fitness value $f_{3}(v)$ as the reciprocal of the standard deviation by the idle time of each station for balancing the workload of each station. $t_{k}{ }^{\mathrm{W}}$ is the total processing time of each station. Then we give the adaptive weights for each fitness, where $w_{1}+w_{2}+w_{3}=1$. The special defination of fitness function is showed as follows:

$$
f(v)=w_{1}\left(f_{1}(v)-z_{1}^{\min }\right)+w_{2}\left(f_{2}(v)-z_{2}^{\min }\right)+w_{3}\left(f_{3}(v)-z_{3}^{\min }\right)
$$

where

$$
\begin{aligned}
& f_{1}(v)=\frac{1}{C_{T}(v)} \\
& f_{2}(v)=n_{C}(v) \\
& f_{3}(v)=\frac{1}{m-1} \sum_{k=1}^{m}\left(\left(C_{T}(v)-t_{k}^{W}(v)\right)-\frac{1}{m} \sum_{j=1}^{m}\left(C_{T}(v)-t_{j}^{W}(v)\right)\right)
\end{aligned}
$$

\section{Local Search Procedure}

Two individuals with high fitness values are likely to have dissimilar machine assignment and operation sequences, and the recombination may result in offspring of poor performance. This means that the genetic operations by themselves have limited ability in finding the global optimal solution. Many researchers have found that the convergence speed of simple GAs is relatively slow $^{(22)}$. One promising approach for improving the convergence speed to the global optimum is the use of local search in GAs ${ }^{(27)}$. In this study, two local search methods are proposed to enhance the search ability of GA. Both the two local search methods are based on critical stations in order to improve their effectiveness and efficiency.

4.1 Task Sequence Search Let $s(i)$ be the station to which task $i$ is assigned. First we introduce the logical function 
Table 2. Performance of the proposed algorithm

\begin{tabular}{|c|c|c|c|c|c|c|c|c|c|c|c|}
\hline \multicolumn{3}{|c|}{ Test Problems } & \multicolumn{3}{|c|}{ Cycle Time $\left(C_{T}\right)$} & \multicolumn{3}{|c|}{ Test Problems } & \multicolumn{3}{|c|}{ Cycle Time $\left(C_{T}\right)$} \\
\hline $\begin{array}{l}\text { \# of } \\
\text { tasks }\end{array}$ & $\begin{array}{c}\text { \# of } \\
\text { stations }\end{array}$ & $\begin{array}{c}\text { WEST } \\
\text { ratio }\end{array}$ & $\begin{array}{c}\text { Levitin et al.'s } \\
\text { Recursive } \\
\end{array}$ & $\begin{array}{l}\text { Levitin et al.'s } \\
\text { Consecutive }\end{array}$ & $\begin{array}{r}\text { Proposed } \\
\text { approach } \\
\end{array}$ & $\begin{array}{l}\text { \# of } \\
\text { tasks }\end{array}$ & $\begin{array}{c}\text { \# of } \\
\text { stations }\end{array}$ & $\begin{array}{c}\text { WEST } \\
\text { ratio }\end{array}$ & $\begin{array}{l}\text { Levitin et al.'s } \\
\text { Recursive }\end{array}$ & $\begin{array}{l}\text { Levitin et al.'s } \\
\text { Consecutive }\end{array}$ & $\begin{array}{l}\text { Proposed } \\
\text { approach } \\
\end{array}$ \\
\hline \multirow[t]{4}{*}{25} & 3 & 8.33 & 518 & 503 & 503 & \multirow{4}{*}{89} & 8 & 11.13 & 638 & 505 & 494 \\
\hline & 4 & 6.25 & 351 & 330 & 327 & & 12 & 7.42 & 455 & 371 & 370 \\
\hline & 6 & 4.17 & 343 & 234 & 213 & & 16 & 5.56 & 292 & 246 & 236 \\
\hline & 9 & 2.78 & 138 & 125 & 123 & & 21 & 4.24 & 277 & 209 & 205 \\
\hline \multirow[t]{4}{*}{35} & 4 & 8.75 & 551 & 450 & 449 & \multirow[t]{4}{*}{111} & 9 & 12.33 & 695 & 586 & 557 \\
\hline & 5 & 7.00 & 385 & 352 & 344 & & 13 & 8.54 & 401 & 339 & 319 \\
\hline & 7 & 5.00 & 250 & 222 & 222 & & 17 & 6.53 & 322 & 257 & 257 \\
\hline & 12 & 2.92 & 178 & 120 & 113 & & 22 & 5.05 & 265 & 209 & 192 \\
\hline \multirow[t]{4}{*}{53} & 5 & 10.60 & 903 & 565 & 554 & \multirow[t]{4}{*}{148} & 10 & 14.80 & 708 & 638 & 600 \\
\hline & 7 & 7.57 & 390 & 342 & 320 & & 14 & 10.57 & 537 & 441 & 427 \\
\hline & 10 & 5.30 & 35 & 251 & 230 & & 21 & 7.05 & 404 & 325 & 300 \\
\hline & 14 & 3.79 & 243 & 166 & 162 & & 29 & 5.10 & 249 & 210 & 202 \\
\hline \multirow[t]{4}{*}{70} & 7 & 10.00 & 546 & 490 & 449 & \multirow[t]{4}{*}{297} & 19 & 15.63 & 1129 & 674 & 646 \\
\hline & 10 & 7.00 & 313 & 287 & 272 & & 29 & 10.24 & 571 & 444 & 430 \\
\hline & 14 & 5.00 & 231 & 213 & 204 & & 38 & 7.82 & 442 & 348 & 344 \\
\hline & 19 & 3.68 & 198 & 167 & 154 & & 50 & 5.94 & 363 & 275 & 256 \\
\hline
\end{tabular}

$W_{i}(k)$ which return false if task $i$ cannot be transferred from station $s(i)$ to station $\mathrm{k}$, and true otherwise:

$$
\begin{aligned}
& W_{i}(k)=\text { false if } \\
& \quad s(i)>k \text { and } \exists j \in \operatorname{suc}(i)>k, \text { or } \\
& \\
& s(i)>k \text { and } \exists j: i \in \operatorname{suc}(j), s(j)>k \\
& W_{i}(k)=\text { true, otherwise. }
\end{aligned}
$$

For a pair of tasks $i, j$, if

$$
W_{i}(s(j))=W_{j}(s(i))=\operatorname{true} \wedge i \notin \operatorname{pre}(i) \wedge j \notin \operatorname{pre}(i)
$$

then, the two tasks are said to be exchangeable. Let $R(i)$ be the robot which is allocated for station $s(i)$, and $T_{l}$ be the total assembly time of station $l$. For a critical task $i$ and task $j$ which is not in $s(i)$, if

$$
t_{j, s(i)}<t_{i, s(i)} \text { and } T_{s(j)}-t_{j, s(j)}+t_{i, s(j)}<C_{T}
$$

then the exchange between tasks $i$ and $j$ is called worthwhile. The task sequence search is as follows:

step 1 . Decode the chromosome and identify a critical station $l^{*}$; step 2. For a task $i$ that assigned to the critical station $l^{*}$, look for a task $j$ that is not assigned to station $l^{*}$, and is exchangeable with task $i$.

step 3. Repeat Step 2 until the exchange between tasks $i$ and $j$ is worthwhile or all the tasks in the critical station are tried.

step 4. Repeatedly perform the above search steps until the chromosome cannot be improved any more.

4.2 Robot Assignment Search Let $N(i)$ denote the set of machine assignment neighborhood of solution $i$. The enlarged two-pace machine assignment neighborhood is defined as the union of the neighborhood of each robot assignment neighbor of solution $i$

$$
N^{2}(i)=\cup_{j \in N(i)} N(j)
$$

During the robot assignment search, the local search will implement over two-pace neighborhood when it reaches the local optima of one-pace neighborhood, and is called two-pace robot assignment search. During the robot assignment search, when the local optima of two-pace robot assignment neighborhood is reached, the neighbors of the two-pace local optima are improved by the task sequence search to help the robot assignment search escape from the local optima. Since the size of the two-pace neighborhood is very large and the task sequence search is very computationally complex, to improve each neighbor solution by task sequence search needs a great computation time. Therefore, only those neighbor solutions that are local optimums of the two-pace robot assignment neighborhood are improved by task sequence search so as to save computation time.

\section{Numerical Experiments}

In the literature, no benchmark data sets are available for rALB. We collect 8 representative precedence graphs from ${ }^{(29)}$, which are widely used in the sALB-1 literature ${ }^{(30)}$. These precedence graphs contain with 25-297 tasks. From each precedence graph, 4 different rALB-2 problems are generated by using different WEST ratios: 3, 5, 7, 10, 15. WEST ratio, as defined by Dar-EI ${ }^{(31)}$, measures the average number of activities per station. For each problem, the number of station is equal to the number of robots, and each task can be processed on any robot. The task time data

are generated at random, while two statistical dependence are maintained: (1) statistical dependence of task times on the task type, (2) statistical dependence of task times on the robot on which the task is processed.

To validate the effectiveness of our hybrid GA, we compare our approach with Levitin et al.'s two algorithms. Recently, Levitin et $a l .{ }^{(26)}$ develop an efficient approaches for rALB problems. It is similar research with our purpose, aims to achieve a balanced distribution of work between different stations (balance the line) while assigning to each station the robot best fit for the activities assigned to it. Levitin et al. proposed two algorithms named as recursive assignment method and consecutive assignment method. If we look each robot as one type and deleting this type from the total types of robots when the robot is allocated for a station, Levitin et al.'s two algorithms can be adapted to solve the 32 rALB-2 problems here. Hence, the two algorithms proposed by Levitin et al. are also used to solve the 32 problems.

All the simulation experiments were performed on Pentium 4 processor (2.6-GHz clock). The adopted parameters of the hGA are listed as following: maximal generations maxGen $=1000$; population size popSize $=100$; exhange crossover probability 


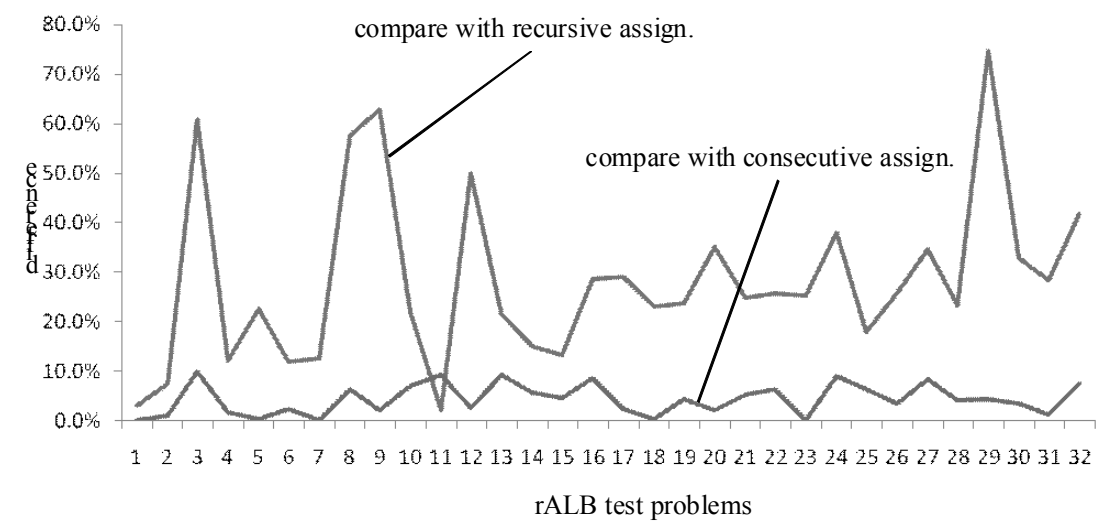

Fig. 10. The difference between different approaches

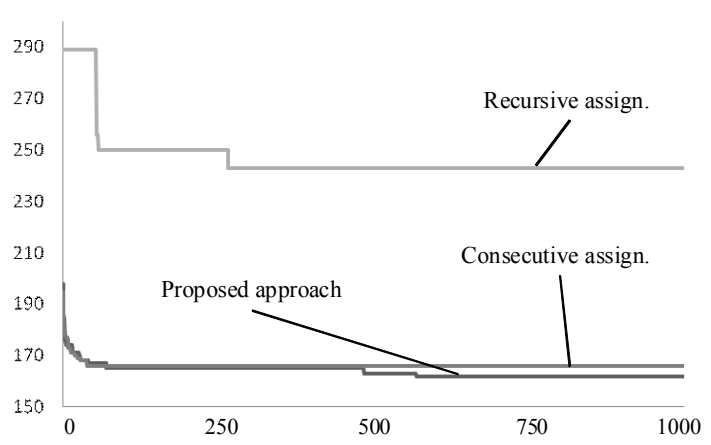

(a) test $35-12$

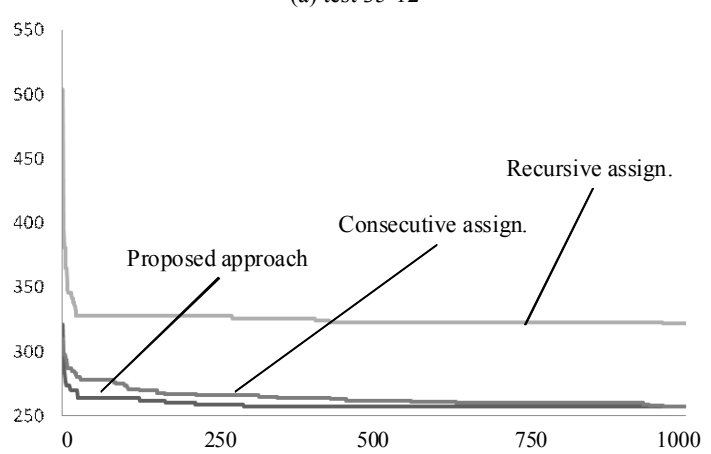

(b) test 111-17

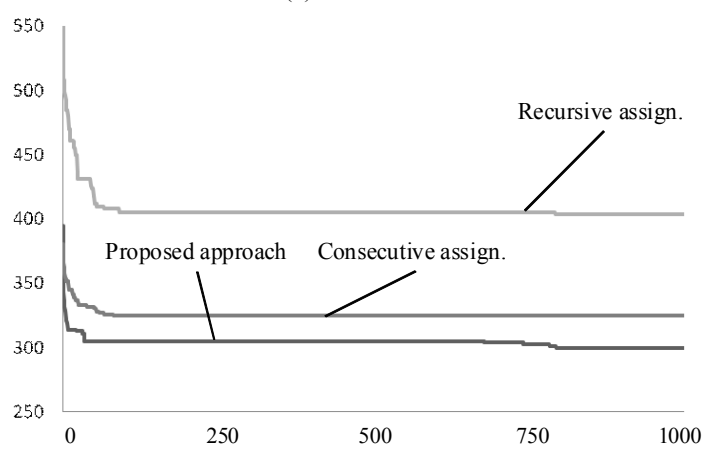

(c) test $148-21$

Fig. 11. Evolutionary process of 3 different approaches

$p_{C}=0.20$; mixed crossover probability $p_{M}{ }^{1}=0.80$; task sequence mutation probability $p_{M}^{2}=0.05$; robot assign. mutation probability $p_{M}{ }^{3}=0.10$. Levitin et al.'s two algorithms used the same population size and maximal generations as the hGA.
Table 2 shows the experiment results of Levitin et al.'s two algorithms and proposed approach for 32 different scale test problems. As depicted in Table 2, all of results by proposed approach are obvious better than Levitin et al.'s recursive assignment method. And most results of proposed approach are better than and Levitin et al.'s consecutive assignment method, except to the test $25-3$, test $35-7$ and test 111-17. The differences of 32 test problems between proposed approach with Levitin et al.'s approaches are showed in Fig. 10.

To clearly see the convergence performance of proposed approach, we give the evolutionary process of proposed approach and Levitin et al.'s two methods by combining 3 different scale test problems: small-scale test 35-12, middle-scale test 111-17 and large-scale test 148-21. The evolutionary processes are showed in Fig. 11. As depicted in Fig. 11, the convergence of proposed approach are faster than each of other approaches. The difference of convergence increased with increasing the test problems size. Furthermore, though Levitin et al.'s consecutive found the same result with proposed approach for test 111-17, but the convergence speed of proposed approach was faster. The simulations show that propose hybrid GA with combining local search approach is computationally efficient and effective to find the best solution and convergence speed.

\section{Conclusions}

Robotic assembly line has been an important manufacturing system in the modern era. The objective of this work is to develop an efficient solution for the robotic assembly line balancing problem. This solution aims to achieve a balanced distribution of work between different stations and assign to each station the robot best fit for the activities assigned to it. The result of such solution would be an increased production rate of the line.

A new representation method adapting the GA to the rALB-II problem was proposed. Advanced genetic operators adapted to the specific chromosome structure and the characteristics of the rALB problem were used. In order to strengthen the search ability, two kinds of local search were integrated under the framework the genetic algorithm. The coordination among the three kinds of local search was well considered. The neighborhood structure of the local search can be adjusted dynamically. The balance between genetic search and local search was also investigated. The performance of proposed method was validated through simulation experiments. The simulation showed that our algorithm is computationally efficient and effective to find the best solution. 
The solution obtained by our algorithm outperforms the results from previous works.

(Manuscript received Jun. 1, 2007, revised Oct. 25, 2007)

\section{References}

(1) W. B. Helgeson, M. E. Salveson, and W. W. Smith : "How to balance an assembly line", Technical Report,Carr Press, New Caraan, Conn. (1954)

(2) A. Scholl : "Balancing and Sequencing of Assembly Lines", Physica-Verlag, Heidelberg. (1999)

(3) Y. K. Kim, Y. J. Kim, and Y. H. Kim : "Genetic algorithms for assembly line balancing with various objectives", Computers \& Industrial Engineering, Vol.30, No.3, pp.397-409 (1996)

(4) A. L. Gutjahr and G. L. Nemhauser : "An algorithm or the line balancing problem, Management Science”, Vol.11, pp.308-315 (1964)

(5) E. Falkenauer and A. Delchambre : "A genetic algorithm for bin packing and line balancing", In the Proceedings of the 1992 IEEE International Conference on Robotics and Automation, Nice, France, pp.1189-1192 (1992)

(6) E. J. Anderson and M. C. Ferris : "Genetic algorithms for combinatorial optimization: the assemble line balancing problem", ORSA Journal on Computing, 6: pp.161-173 (1994)

(7) J. Rubinovitz and G. Levitin : "Genetic algorithm for assembly line balancing”, International Journal of Production Economics, Vol.41, pp.343-354 (1995)

(8) M. Gen, Y. Tsujimura, and Y. Li : "Fuzzy assembly line balancing using genetic algorithms", Computers \& Industrial Engineering, Vol.31, No.3/4, pp.631-634 (1996)

(9) J. Bautista, R. Suarez, M. Mateo, and R. Companys : "Local search heuristics for the assembly line balancing problem with incompatibilities between tasks", In the Proceedings of the 2000 IEEE International Conference on Robotics and Automation, San Francisco, CA, pp.2404-2409 (2000)

(10) I. Sabuncuoglu, E. Erel, and M. Tanyer : "Assembly line balancing using genetic algorithms", Journal of Intelligent Manufacturing, Vol.11, No.3, pp.295-310 (2000)

(11) J. F. Goncalves and J. R. Almeida : "A hybrid genetic algorithm for assembly line balancing", Journal of Heuristics, Vol.8, No.6, pp.629-642 (2002)

(12) E. C. Brown and R. T. Sumichrast : "Evaluating performance advantages of grouping genetic algorithms", Engineering Applications of Artificial Intelligence, Vol.18, pp.1-12 (2005)

(13) A. C. Nearchou: "Balancing large assembly lines by a new heuristic based on differential evolution method", International Journal of Advanced Manufacturing Technology, Vol.34, No.9-10, pp.1016-1029 (2006)

(14) J. Rubinovitz and J. Bukchin : "Design and balancing of robotic assembly lines", In Proceedings of the Fourth World Conference on Robotics Research, Pittsburgh, PA (1991)

(15) J. Roubinovitz and J. Bukchin : "RALB-a heuristic algorithm for design and balancing of robotic assembly line", Annals of the CIRP, Vol.42, pp.497-500 (1993)

(16) J. Bukchin and M. Tzur : "Design of flexible assembly line to minimize equipment cost”, IIE Transactions, Vol.32, pp.585-598 (2000)

(17) D. M. Tsai and M. J. Yao : "A line-balanced-base capacity planning procedure for series-type robotic assembly line", International Journal of Production Research, Vol.31, pp.1901-1920 (1993)

(18) H. Kim and S. Park : "Strong cutting plane algorithm for the robotic assembly line balancing", International Journal of Production Research, Vol.33, No.8, pp.2311-2323 (1995)

(19) M. Khouja, D. E. Booth, M. Suh, and Jr. J. K. Mahaney : "Statistical prodecures for task assignment and robot selection in assembly cells", International Journal of Computer Integrated manufacturing, Vol.13, No.2, pp.95-106 (2000)

(20) G. Nicosia, D. Paccarelli, and A. Pacifici : "Optimally balancing assembly lines with different workstations", Discrete Applied Mathematics, Vol.118, pp.99-113 (2002)

(21) J. Gao and M. Gen : "A Hybrid Genetic Algorithm for Robotic Assembly Line Balancing”, Proc. of SOPEJ, pp.46-54 (2005)

(22) M. Gen and R. Cheng : "Genetic Algorithms \& Engineering Optimization", New York: Wiley (2000)

(23) Y. Tsujimura, M. Gen, and E. Kubota : "Solving fuzzy assembly-line balancing problem with genetic algorithms", Computers \& Industrial Engineering, Vol.29, No.1-4, pp.543-547 (1995)

(24) J. Rubinovitz and G. Levitin : "Genetic algorithm for line balancing", Int. J. Production Economics, Vol.41, pp.343-354 (1995)
(25) M. Gen and R. Cheng : "Genetic Algorithms \& Engineering Design", New York: Wiley (1997)

(26) G. Levitin, J. Rubinovitz, and B. Shnits : "A genetic algorithm for robotic assembly line balancing", European Journal of Operational Research, Vol.168, No.3, pp.811-825 (2006)

(27) N. Krasnogor and J. Smith : "A mimetic algorithm with self-adaptive local search: TSP as a case study", Proc. of 2000 Genetic and Evolutionary Computation Conference, July 10-12, Las Vegas, NV, pp.987-994 (2000)

(28) P. Moscato and M. Norman : "A mimetic approach for the traveling salesman problem: implementation of a computational ecology for combinatorial optimization on message-passing systems", Proceedings of the International Conference on Parallel Computing and Transputer Applications, Amsterdam (1992)

(29) Online: http://www.assembly-line-balancing.de/

(30) A. Scholl : Data of Assembly Line Balancing Problems. Schriften zur Quantitativen Betriebswirtschaftslehre 16/93, Th Darmstadt (1993)

(31) E. M. Dar-EI (Mansoor) : "MALB-a heuristic technique for balancing large single-model assembly lines", AIIE Transactions, Vol.34, pp.343-356 (1973)

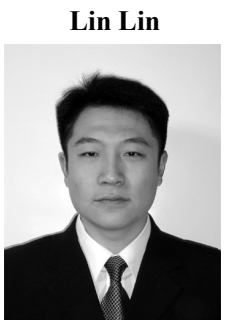

(Student Member) graduated from Shenyang Yingcai College majored in computer engineering, Shenyang, China in 1999, and graduated his master's degree from Graduate Schoole of IPS, Waseda Univ. in 2005. After that, he is a Ph.D candidate and Research Assistant at Waseda Univ. He concentrates his academic interests on Genetic Algorithms, Multiobjective Optimization, Neural networks, and the applications of evolutionary techniques on Network Design, Reliability of Communication Network, and Logistics Optimization etc.

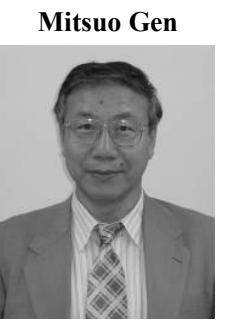

(Member) received the Ph.D. degree from the Kogakuin University, Japan in 1974. He was a lecturer in 1974-1980, an Assoc. professor in 1980-1987, professor in 1987-2003 at Ashikaga Institute of Technology. He has been a professor in IPS, Waseda Univ. since Apr. 2003. He was a visiting Assoc. professor at University of Nebraska Lincoln, USA in 1981-1982, a visiting professor at University of California at Berkeley, USA from Aug. 1999 to Mar. 2000. His research interests include Genetic Algorithms, Neural Network, Fuzzy Logic, and the applications of evolutionary computation approaches to Network Design, Scheduling and System Reliability Design etc. He has authored several books, such as Genetic Algorithms \& Engineering Design, John Wiley \& Sons, New York (1997), Genetic Algorithms \& Engineering Optimization, John Wiley \& Sons, New York (2000) with Dr. Runwei Cheng, and is an area editor of the journal Computers \& Industrial Engineering.

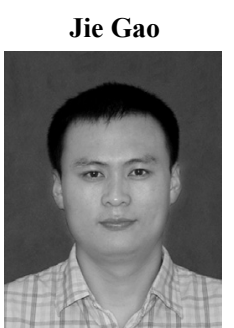

(Non-member) is an assistant professor of the Department of Industrial Engineering, Xian Jiaotong University, China from 2007. He received the combined B.S. degree in Industrial Engineering and Mechanics in 2001, and PhD degree in Management Science and Engineering in 2007 all from Xian Jiaotong University. He was an exchange student at Graduate School of Information, Production \& Systems, Waseda University, Japan from Oct. 2004 to Mar. 2006. His main research interests include Maintenance Scheduling, flexible Job-shop Scheduling, Assembly Line Balancing, Evolutionary Algorithms and Service Management. 